\title{
Field theory for charged fluids and colloids
}

\author{
Roland R. Netz ${ }^{\$}$ and Henri Orland* \\ \$Max-Planck-Institut für Kolloid- und Grenzflächenforschung, Kantstr. 55, 14513 Teltow, Germany \\ * Service de Physique Théorique, CEA-Saclay, 91191 Gif sur Yvette, France
}

(June 20, 2021)

\begin{abstract}
A systematic field theory is presented for charged systems. The one-loop level corresponds to the classical Debye-Hückel (DH) theory, and exhibits the full hierarchy of multi-body correlations determined by pair-distribution functions given by the screened DH potential. Higher-loop corrections can lead to attractive pair interactions between colloids in asymmetric ionic environments. The free energy follows as a loop-wise expansion in half-integer powers of the density; the resulting two-phase demixing region shows pronounced deviations from DH theory for strongly charged colloids.
\end{abstract}

Since the early work of Debye and Hückel (DH) it is known that electrostatic interactions in a mixture of positively and negatively charged particles produce a net attraction [1]. This is due to charge screening: Each charge is (on average) predominantly surrounded by oppositely charged particles, which thus leads to an overall attraction between the particles. The resulting DH free energy contribution has been theoretically demonstrated to lead to phase separation in the context of ionic fluids [2] and colloidal mixtures [3], in agreement with experimental [4] and numerical [5] work on ionic fluids and experiments on colloidal mixtures [6]. The exact nature and origin of the $\mathrm{DH}$ term has remained somewhat unclear, and several improvements have been devised based on series expansions and liquid-state theory [7], explicit incorporation of dipole pairs [2], and density-functional theory [3].

A second intensely debated question concerns the possible existence of attractive interactions between similarly charged objects in electrolyte solution [8]. Experimentally, such an attraction has been seen for DNA 9] and strongly charged microspheres which are confined between charged plates [10]. Clearly, the phase separation observed for colloidal mixtures [6] is a priori not an indication for such an attractive interaction, because the dense phase is induced by attractions between oppositely charged particles, as becomes explicit within DH theory (also, see the discussion in [3]).

In this article we present a systematic field theory for charged systems, and calculate both the free energy and the effective interactions between charged particles immersed in an electrolyte solution. At the one-loop level, we recover the classical DH theory, the nature of which transpires in an especially lucid fashion within our framework: we find the full hierarchy of multi-body correlations to be present, with all pair-distribution functions given by the screened DH interaction. This means in specific that triplet correlations are already included at the DH level (in contrast to implicit assumptions in recent theories [2]), and that effective interactions between similarly charged particles are repulsive. At higher order in our theory (which corresponds to including multi- loop diagrams), non-trivial multi-body interactions appear, and, consequently, the multibody correlations acquire contributions which cannot be described as superposition of pair correlations. Also, the effective pair interaction receives corrections which can be attractive if i) the electrolyte is asymmetric and consists of multivalent counterions and monovalent coions, or if ii) the colloidal charge is overcompensated by salt ions. The latter situation is realized in experiments on charged microspheres, where a strong attraction is only found in the vicinity of a charged wall [10]. The free energy of an ionic solution, expanded in the number of loops, follows to be a series in half-integer powers of the density, and thus constitutes a systematic low-density expansion [11]. The effects of higher-loop contributions on the demixing transition become increasingly important for highly charged colloids and lead to pronounced deviations from DH theory.

To proceed, we consider the partition function of $N$ charged, fixed test particles, immersed in a multicomponent electrolyte solution with (in general) $M$ different types of ions,

$$
\begin{aligned}
Z\left[\left\{R_{N}\right\}\right]= & \prod_{j=1}^{M}\left[\frac{1}{n_{j} !} \prod_{k=1}^{n_{j}} \int \frac{\mathrm{d} \mathbf{r}_{k}^{(j)}}{\lambda^{3}}\right] \\
& \exp \left\{-\frac{1}{2} \int \mathrm{d} \mathbf{r} \mathrm{d} \mathbf{r}^{\prime} \hat{\rho}_{c}(\mathbf{r}) v\left(\mathbf{r}-\mathbf{r}^{\prime}\right) \hat{\rho}_{c}\left(\mathbf{r}^{\prime}\right)\right\},
\end{aligned}
$$

where $v(\mathbf{r})=\ell_{B} / r$ is the Coulomb operator and the charge density operator $\hat{\rho}_{c}$ is defined by

$$
\hat{\rho}_{c}(\mathbf{r}) \equiv \sum_{i=1}^{N} Q_{i} \delta\left(\mathbf{r}-\mathbf{R}_{i}\right)+\sum_{j=1}^{M} \sum_{k=1}^{n_{j}} q_{j} \delta\left(\mathbf{r}-\mathbf{r}_{k}^{(j)}\right)
$$

with $Q_{i}$ and $q_{j}$ being the charges (in units of the elementary charge $e$ ) of the test particles and the ions, respectively. The length $\lambda$ is an arbitrary constant, and the Bjerrum length $\ell_{B} \equiv e^{2} / 4 \pi \epsilon k_{B} T$ defines the length at which two unit charges interact with thermal energy $k_{B} T$. Electroneutrality of course requires $\sum_{i=1}^{N} Q_{i}+\sum_{j=1}^{M} n_{j} q_{j}=0$. 
Noting that the inverse Coulomb operator can be explicitly written as $v^{-1}(\mathbf{r})=-\nabla^{2} \delta(\mathbf{r}) / 4 \pi \ell_{B}$, after a HubbardStratonovich transformation, the partition function is given by

$$
Z\left[\left\{R_{N}\right\}\right]=\int \frac{\mathcal{D} \phi}{Z_{0}} \exp \left\{-\frac{1}{8 \pi \ell_{B}} \int \mathrm{d} \mathbf{r}(\nabla \phi)^{2}-i \sum_{i=1}^{N} Q_{i} \phi\left(\mathbf{R}_{i}\right)+\sum_{j=1}^{M} n_{j} \log \left[\int \frac{\mathrm{d} \mathbf{r}}{V} \mathrm{e}^{-i q_{j} \phi(\mathbf{r})}\right]+\mathcal{S}\right\},
$$

where $Z_{0}$ is the partition function of the inverse Coulomb operator, $Z_{0} \sim \operatorname{det} v$, and the entropy of ideal mixing is $\mathcal{S} \equiv-\sum_{j} n_{j} \ln \left(\lambda^{3} c_{j}\right)$ with $c_{j} \equiv n_{j} / V$ denoting the concentration of ion species $j$. Performing a cumulant expansion of (3) in powers of $\phi$, we can rewrite the partition function as

$$
Z\left[\left\{R_{N}\right\}\right]=\int \frac{\mathcal{D} \phi}{Z_{0}} \exp \left\{-\frac{1}{2} \int \mathrm{d} \mathbf{r} \mathrm{d} \mathbf{r}^{\prime} \phi(\mathbf{r}) v_{\mathrm{DH}}^{-1}\left(\mathbf{r}-\mathbf{r}^{\prime}\right) \phi\left(\mathbf{r}^{\prime}\right)-i \sum_{i=1}^{N} Q_{i} \phi\left(\mathbf{R}_{i}\right)+W[\phi]+\mathcal{S}\right\},
$$

where $v_{\mathrm{DH}}$ is determined via the inverse operator equation (the so-called Dyson equation in field theory)

$$
v_{\mathrm{DH}}^{-1}(\mathbf{r}) \equiv v^{-1}(\mathbf{r})+I_{2} \delta(\mathbf{r})
$$

which is solved by the well-known $\mathrm{DH}$ interaction $v_{\mathrm{DH}}(\mathbf{r})=\ell_{B} e^{-r \kappa} / r$ with the screening length $\kappa^{-1}$ defined by $\kappa^{2} \equiv 4 \pi \ell_{B} I_{2}$. All anharmonic terms are contained in the non-local potential $W$, which is up to eighth order given by

$$
\begin{aligned}
W[\phi]= & \frac{i I_{3} V}{3 !} \overline{\phi^{3}}+\frac{I_{4} V}{4 !}\left(\overline{\phi^{4}}-3{\overline{\phi^{2}}}^{2}\right)-\frac{i I_{5} V}{5 !}\left(\overline{\phi^{5}}-10 \overline{\phi^{2}} \overline{\phi^{3}}\right)-\frac{I_{6} V}{6 !}\left(\overline{\phi^{6}}-15 \overline{\phi^{4}} \overline{\phi^{2}}-10{\overline{\phi^{3}}}^{2}+30{\overline{\phi^{2}}}^{3}\right) \\
& +\frac{I_{8} V}{8 !}\left(\overline{\phi^{8}}-28 \overline{\phi^{6}} \overline{\phi^{2}}-56 \overline{\phi^{5}} \overline{\phi^{3}}-35{\overline{\phi^{4}}}^{2}+420{\overline{\phi^{4}}}_{\bar{\phi}^{2}}{ }^{2}+560{\overline{\phi^{2}}}_{\bar{\phi}^{3}}^{2}-630{\overline{\phi^{2}}}^{4}\right) .
\end{aligned}
$$

We have introduced the generalized ionic strength $I_{n}$, which is defined as $I_{n} \equiv \sum_{j=1} q_{j}^{n} c_{j}$ and can take both positive and negative values. In these equations $\overline{\phi^{n}}$ denotes moments of the field, $\overline{\phi^{n}} \equiv \int \mathrm{d} \mathbf{r} \phi^{n}(\mathbf{r}) / V$. The action is invariant with respect to a change of the gauge field $\bar{\phi}$. We therefore set $\bar{\phi}=0$. The linear term in $\phi$ in Eq.(睴) can be removed by a shift of the fluctuating field $\phi$, and the partition function then takes the form

$$
Z\left[\left\{R_{N}\right\}\right]=\exp \left\{\mathcal{S}-\frac{1}{2} \sum_{i, j} Q_{i} Q_{j} v_{\mathrm{DH}}\left(\mathbf{R}_{i}-\mathbf{R}_{j}\right)\right\} \int \frac{\mathcal{D} \phi}{Z_{0}} \exp \left\{-\frac{1}{2} \int \mathrm{d} \mathbf{r} \mathrm{d} \mathbf{r}^{\prime} \phi(\mathbf{r}) v_{\mathrm{DH}}^{-1}\left(\mathbf{r}-\mathbf{r}^{\prime}\right) \phi\left(\mathbf{r}^{\prime}\right)+W[\tilde{\phi}]\right\}
$$

where $\tilde{\phi}(\mathbf{r}) \equiv \phi(\mathbf{r})-i \sum_{i} Q_{i} v_{\mathrm{DH}}\left(\mathbf{r}-\mathbf{R}_{i}\right)$. Up to this point, our calculations are (in principle) exact: keeping terms of all powers in $W$ in (7) leads to a model equivalent to the original partition function (11). They are also systematic, in that keeping terms of higher and higher order of $W$ should make the resulting theory a more and more faithful representation of the underlying physical model. In fact, we will demonstrate that the lowest approximation, where the potential $W$ and thus anharmonic terms in $\phi$ are neglected altogether, is equivalent to the classical DH theory. In this case, it follows from (7) that the dimensionless pair interaction $U_{2}$ between two test particles is just the $\mathrm{DH}$ potential,

$$
U_{2}\left(\mathbf{R}_{1}-\mathbf{R}_{2}\right)=Q_{i} Q_{j} v_{\mathrm{DH}}\left(\mathbf{R}_{1}-\mathbf{R}_{2}\right)
$$

and the two-point correlation function is $g_{2}\left(\mathbf{R}_{1}-\mathbf{R}_{2}\right) \propto$ $e^{-U\left(\mathbf{R}_{1}-\mathbf{R}_{2}\right)}$ with a proportionality constant such that it is normalized [12]. Neglecting $W$, there are no multibody interactions between test particles in (7), and higherorder correlation functions are therefore given by prod- ucts of the pair correlation function, $g_{3}\left(\mathbf{R}_{1}, \mathbf{R}_{2}, \mathbf{R}_{3}\right) \propto$ $g_{2}\left(\mathbf{R}_{1}-\mathbf{R}_{2}\right) g_{2}\left(\mathbf{R}_{2}-\mathbf{R}_{3}\right) g_{2}\left(\mathbf{R}_{1}-\mathbf{R}_{3}\right)$, and so on. This is the superposition principle, known as a postulate from liquid state theory; it is exactly obeyed in DH theory. To connect to liquid state theory, we note that from Eq. (5) one obtains by inversion the integral equation (which in fact holds for any pair interaction $v$ )

$$
v(\mathbf{r})=v_{\mathrm{DH}}(\mathbf{r})+I_{2} \int \mathrm{d} \mathbf{r}^{\prime} v_{\mathrm{DH}}\left(\mathbf{r}^{\prime}\right) v\left(\mathbf{r}-\mathbf{r}^{\prime}\right),
$$

the field-theoretic version of the Ornstein-Zernicke equation. The DH interaction is the exact solution of this integral equation [13. The DH theory therefore contains correlations of all orders, and there is no need to explicitly add higher-order correlations (compare [2]). Improvements can only come from adding non-trivial higher-body effective interactions, i.e., from violations of the superposition principle. This is the effect of the potential $W$, as we will demonstrate in the following. 
Expanding $W[\phi]$ in the exponential of Eq.(7), the first correction comes from the cubic term,

$$
Z\left[\left\{R_{N}\right\}\right] \propto \exp \left\{\mathcal{S}-\frac{1}{2} \sum_{i, j} Q_{i} Q_{j} v_{\mathrm{DH}}\left(\mathbf{R}_{i}-\mathbf{R}_{j}\right)-\frac{I_{3}}{6} \sum_{i, j, k} Q_{i} Q_{j} Q_{k} \Omega_{3}\left(\mathbf{R}_{i}, \mathbf{R}_{j}, \mathbf{R}_{k}\right)\right\}
$$

with the three-point vertex given by $\Omega_{3}\left(\mathbf{R}_{1}, \mathbf{R}_{2}, \mathbf{R}_{3}\right) \equiv \int \mathrm{d} \mathbf{r} v_{\mathrm{DH}}\left(\mathbf{r}-\mathbf{R}_{1}\right) v_{\mathrm{DH}}\left(\mathbf{r}-\mathbf{R}_{2}\right) v_{\mathrm{DH}}\left(\mathbf{r}-\mathbf{R}_{3}\right)$.

The summation over $(i, j, k)$ in (9) is unrestricted. By considering the case where two of the three indices are equal, one obtains a correction to the pair interaction, which reads

$$
\Delta U_{2}(\mathbf{R})=\frac{I_{3} \ell_{B}^{3}\left(Q_{1}^{2} Q_{2}+Q_{1} Q_{2}^{2}\right)}{6} \Xi(R \kappa),
$$

where the function $\Xi$ (which is positive) is determined by the integral $\Xi(x) \equiv \int \mathrm{d} \mathbf{r} e^{-2 r} e^{-|\mathbf{r}-\mathbf{x}|} / r^{2}|\mathbf{r}-\mathbf{x}|=$ $2 \pi\left\{e^{-x}(\ln 3-\Gamma[0, x])+e^{x} \Gamma[0,3 x]\right\} / x$. The asymptotic behavior of $\Xi$ is

$$
\Xi(x) \simeq \begin{cases}-4 \pi \ln x & \text { for } x \ll 1 \\ 2 \pi \ln 3 \frac{e^{-x}}{x} & \text { for } x \gg 1\end{cases}
$$

and thus shows the same asymptotic behavior as the DH repulsion for large separations. When do we expect attractive interactions between likely charged particles? In other words, when does the prefactor in Eq.(10) become negative? If we assume the test particles to be positively charged, the condition for attraction is that the third-order ionic strength $I_{3}$ is negative. Assuming a homogeneous solution of positive macroions with charge $Z$, concentration $c$, and counter ions of valency $z$, the third-order ionic strength is $I_{3}=c Z\left(Z^{2}-z^{2}\right)$ and clearly always positive: similarly charged particles at finite concentration do not attract each other, in agreement with experiments. On the other hand, considering two single charged macroions, it is easy to see that $I_{3}$ is negative if the salt solution is asymmetric: for positive macroions, attraction is therefore possible i) if one has an electrolyte consisting of negative ions with a higher valency than the positive ions or ii) if there are more negative than positive ions in the local environment (as in experiments between two charged walls 10]). Comparing the strength of the DH repulsion Eq.(8) and the attraction Eq.(10) at large separation we find the attraction to dominate for $c \ell_{B}^{3} Z^{2}>9\left(m^{2}+1\right) /\left(m^{3}-1\right)^{2} \pi \ln ^{2} 3$ for the case of a $m: 1$ electrolyte [14]. Experimentally, it is well-known that asymmetric salts like Calciumchloride induce the precipitation of negatively charged macroions and negatively charged polymers [9].

The phase behavior of charged colloidal mixtures follows from the free energy with all particle coordinates integrated over,

$$
\mathcal{F}=-\ln Z=-\mathcal{S}-\ln \left[\frac{Z_{2}}{Z_{0}}\right]-\ln \left\langle e^{W[\phi]}\right\rangle .
$$

The DH partition function is $Z_{2} \sim \operatorname{det} v_{\mathrm{DH}}$, whereas higher-order correlations are contained in $W$. The expectation value in (12) is evaluated with the $\mathrm{DH}$ propagator. We first evaluate the DH free energy,

$$
f_{\mathrm{DH}} \equiv-\frac{a^{3}}{V} \log \left[\frac{Z_{2}}{Z_{0}}\right]=-\left(\frac{a}{2 \pi}\right)^{3} \int \mathrm{d} \mathbf{q} \log \sqrt{\frac{q^{2}}{q^{2}+\kappa^{2}}}
$$

where the momentum integral goes over a cube of length $2 \pi / a$. Since the integrand is isotropic we distort the integration volume to a sphere and obtain after a straightforward integration

$$
f_{\mathrm{DH}}=-\frac{a^{3} \kappa^{3}}{6 \pi^{2}} \arctan \left[\frac{\pi}{a \kappa}\right]+\frac{a^{2} \kappa^{2}}{6 \pi}+\frac{\pi}{12} \log \left[1+\frac{a^{2} \kappa^{2}}{\pi^{2}}\right] .
$$

In the limit $a \rightarrow 0$ one obtains the well-known result $f_{\mathrm{DH}} \simeq-a^{3} \kappa^{3} / 12 \pi$ (plus corrections which scale linearly in $\kappa^{2}$ and thus correspond to an unimportant shift in the chemical potential). Our corrections as a function of the cut-off take in an approximate fashion the finite ion-sizes into account. The free energy contribution $\Delta f \equiv-\frac{a^{3}}{V} \ln \left\langle e^{W[\phi]}\right\rangle$ is, using Eq.(6), given by

$$
\Delta f=\frac{a^{3} I_{3}^{2}}{12}\left[\chi_{3}+\frac{3}{2}\left\langle\phi^{2}\right\rangle^{2} \chi_{1}\right]-\frac{a^{3} I_{4}^{2}}{48} \chi_{4}+\frac{a^{3} I_{5} I_{3}}{32}\left\langle\phi^{2}\right\rangle^{3} \chi_{1} .
$$

The expectation value $\left\langle\phi^{2}\right\rangle$ is

$$
\left\langle\phi^{2}\right\rangle=\frac{\ell_{B}}{2 \pi^{2}} \int \frac{\mathrm{d} \mathbf{q}}{q^{2}+\kappa^{2}}=\frac{2 \ell_{B}}{a}\left[1-\frac{a \kappa}{\pi} \arctan \left(\frac{\pi}{a \kappa}\right)\right]
$$

and the generalized susceptibility $\chi_{n}$ is defined as

$$
\chi_{n} \equiv \int \mathrm{d} \mathbf{r}\left\langle\phi_{0} \phi_{r}\right\rangle^{n}=4 \pi \ell_{B}^{n}(n \kappa)^{n-3} \Gamma[3-n, a n \kappa] .
$$

Naive scaling predicts the generalized susceptibilities in $\Delta f$ to scale like $\chi_{n} \sim c^{(n-3) / 2}$ as a function of the ion density $c$. Since the dominant terms in $\Delta f$ scale as $I_{n}^{2} \chi_{n}$, one would thus obtain a systematic free-energy expansion in half-integer powers of the density, starting with the DH term $f_{\mathrm{DH}}$, which asymptotically scales as $c^{3 / 2}$ [11]. In practice, the integrals in $\chi_{n}$ diverge in the ultraviolet and thus depend on the ion radius $a$ in a crucial way, which leads to changes from the naive scaling picture. We now present results for the simplified case of colloids of charge $Z$ and concentration $c$ with counterions of valency $z$. Introducing the energy scale $\epsilon \equiv z Z \ell_{B} / a$ and the total ion volume fraction $\tilde{c} \equiv a^{3} c(1+Z / z)$ the $\mathrm{DH}$ theory (which 
amounts to neglecting the term $\Delta f$ ) predicts a critical point at $\epsilon \simeq 5.63$ and $\tilde{c} \simeq 0.0418$, independent of the colloid charge $Z$. Including higher-order terms contained in $\Delta f$ the critical interaction strength $\epsilon$ and volume fraction $\tilde{c}$ depend on $Z / z$, as shown in Fig. 1. The limiting values for $Z / z=1$ are $\epsilon \simeq 5.61$ and $\tilde{c} \simeq 0.0416$ and are thus very similar to the $\mathrm{DH}$ case [15]. For $Z / z>1$ the deviations from DH theory are pronounced. In Fig. 2 we show coexistence curves for $Z / z=1,2$, and 10 (solid, broken, and dotted lines, respectively). The coexistence curve as predicted by DH theory would be indistinguishable from the solid line.

In summary, we introduced a systematic field theory to describe charged colloidal suspensions, including fluctuations of and correlations between charged particles. Attractive colloidal interactions are predicted for asymmetric electrolyte solutions, as realized for multivalent $m: 1$ salt solutions and in the neighborhood of charged walls. A critical point of demixing occurs even in the absence of attractive colloidal interactions, whose location in the temperature-density plane depends strongly on the colloidal charge. The hard-core repulsion between colloids and ions has been incorporated by a small-distance cutoff, similar to recent lattice theories [16. This is a rather poor description of colloidal systems, because here a large size difference between colloids and ions exists. We hope to treat size asymmetries more accurately in the future using perturbative treatments of the hard-core interactions between all particle pairs.

[1] P.W. Debye and E. Hückel, Z. Phys. 24, 185 (1923); see also Ref.[2] in 2].

[2] M.E. Fisher and Y. Levin, Phys. Rev. Lett. 71, 3826 (1993).

[3] R. van Roij and J.-P. Hansen, Phys. Rev. Lett. 79, 3082 (1997).

[4] J.M.H. Levelt Sengers and J.A. Given, Mol. Phys. 80, 899 (1993).
[5] J.P. Valleau, J. Chem. Phys. 95, 584 (1991).

[6] B.V.R. Tata, M. Rajalakshmi, and A.K. Arora, Phys. Rev. Lett. 69, 3778 (1992).

[7] G.R. Stell, K.C. Wu, and B. Larsen, Phys. Rev. Lett. 37, 1369 (1976).

[8] N. Grønbech-Jensen, R.J. Mashl, R.F. Bruinsma, and W.M. Gelbart, Phys. Rev. Lett. 78, 2477 (1997); B.-Y. Ha and A.J. Liu, ibid. 79, 1289 (1997); K.S. Schmitz, Langmuir 13, 5849 (1997).

[9] V.A. Bloomfield, Biopolymers 31, 1471 (1991).

[10] G.M. Kepler and S. Fraden, Phys. Rev. Lett. 73, 356 (1994); J.C. Crocker and D.G. Grier, ibid. 77, 1897 (1996); A.E. Larsen and D.G. Grier, Nature 385, 231 (1997).

[11] Note that the ordinary virial expansion method fails for ionic fluids, as shown in D.A. McQuarrie, Statistical Mechanics (Harper \& Row, New York, 1976), Chap. 15.

[12] It is easily seen that a small-distance cutoff $a$ is needed in order to make the correlation function normalizable. We interpret this cutoff as an effective hard-core radius.

[13] A second interpretation of the DH interaction is obtained by rewriting Eq. (5) in matrix notation as $v_{\mathrm{DH}}=$ $v\left[\delta+I_{2} v\right]^{-1} \simeq v-I_{2} v^{2}+I_{2}^{2} v^{3}-I_{2}^{3} v^{4}+\cdots$. The $\mathrm{DH}$ interaction thus is the renormalized interaction which results from all possible combinations of indirect interaction terms mediated by salt ions.

[14] An effective attractive interactions does not necessarily mean coagulation of particles, since the centrifugal potential (which is logarithmic) has to be overcome.

[15] MC estimates of critical parameters for $Z / z=1$ range from $\tilde{c} \simeq 0.07, \epsilon \simeq 14.3[$ [5] to $\tilde{c} \simeq 0.03, \epsilon \simeq 17.5$ [2]; we attribute the discrepancy to our results, especially in $\epsilon$, to our approximate way of including the hardcore repulsion. The improved DH theory yields $\tilde{c} \simeq 0.028$ and $\epsilon \simeq 17.5$ 2.

[16] I. Borukhov, D. Andelman, and H. Orland, Phys. Rev. Lett. 79, 435 (1997).

FIG. 1. Critical interaction strength $\epsilon \equiv z Z \ell_{B} / a$ (solid line) and critical volume fraction $\tilde{c} \equiv a^{3} c(1+Z / z)$ (broken line) as a function of the colloid/counterion charge ratio $Z / z$.

FIG. 2. Two-phase coexistence regions for $Z / z=1$ (solid line), 2 (broken line), and 10 (dotted line) as a function of volume fraction $\tilde{c}$ and rescaled temperature $z / Z \epsilon$. 
Fig. 1

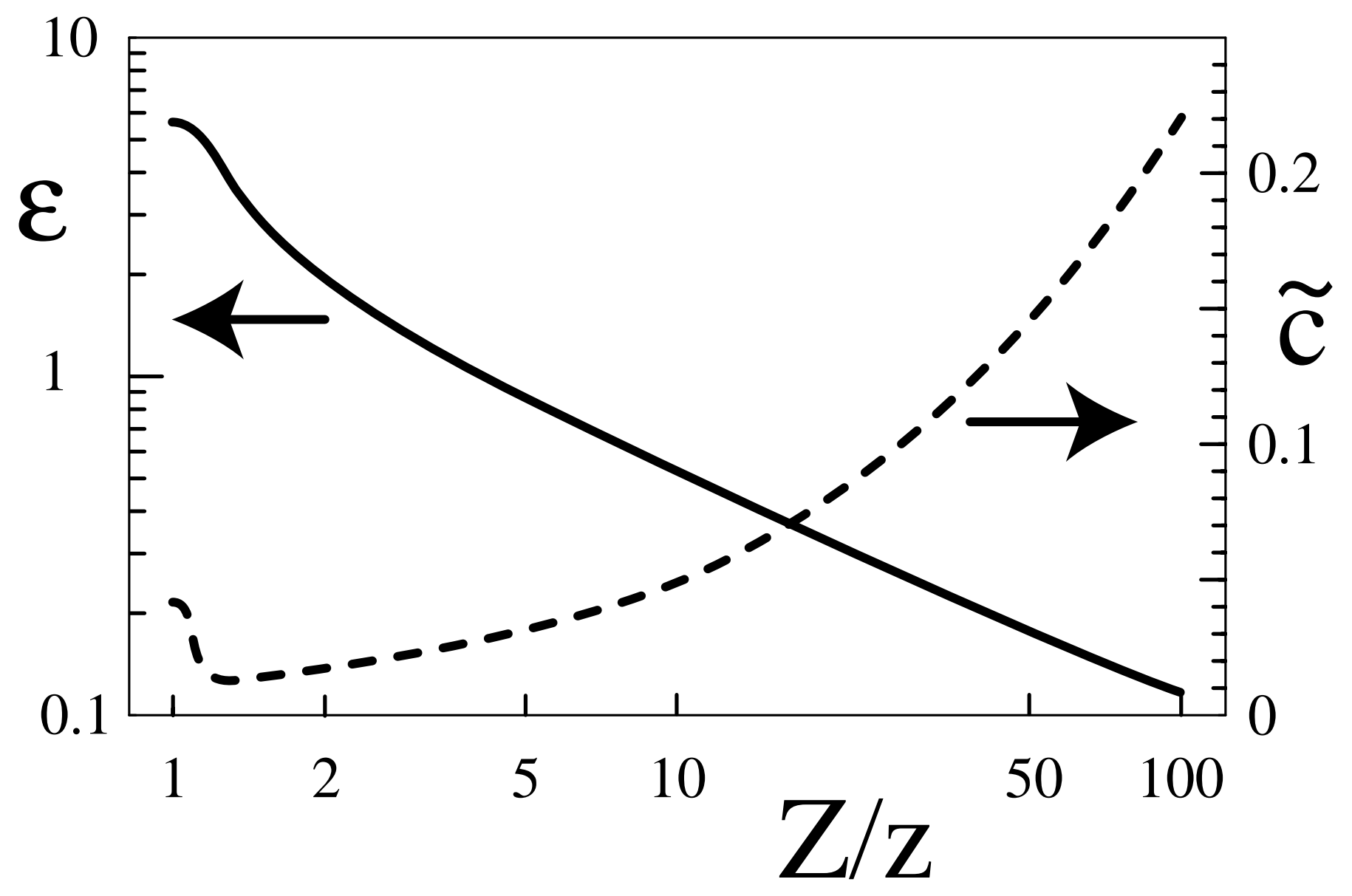


Fig. 2

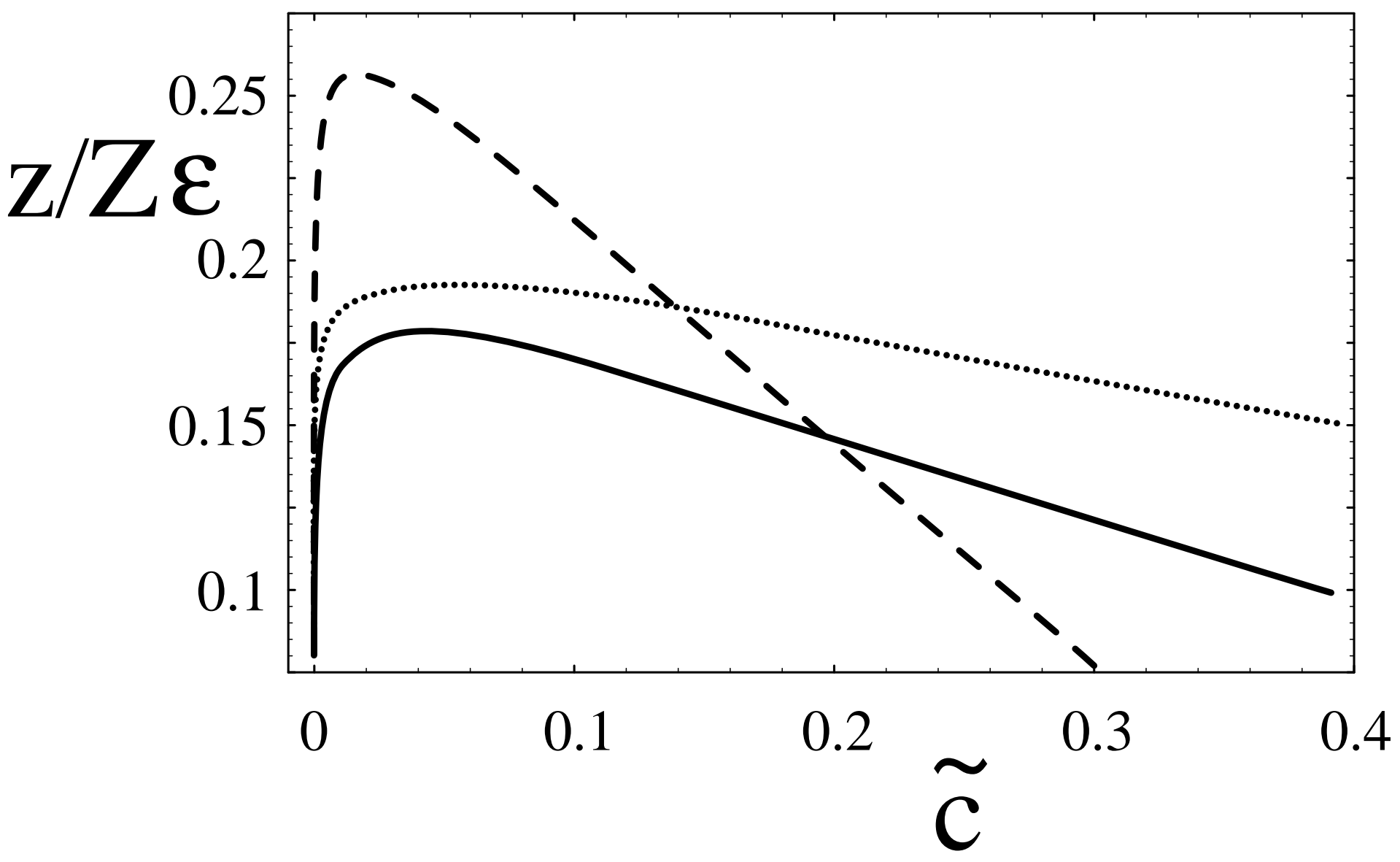

